\title{
Improvement of Hair Graying during a Treatment of Male Pattern Hair Loss Using 1,927-nm Fractionated Thulium Laser Energy and Polydeoxyribonucleotide Injections
}

\author{
Yoon Jin Choi ${ }^{1}$ \\ Suhyun $\mathrm{Cho}^{1}$ \\ Young Koo Kim² \\ Dae Suk Kim³
}

${ }^{1}$ Yonsei BB Skin Clinic, Seoul, Korea
${ }^{2}$ Yonsei Star Skin \& Laser Clinic, Seoul, Korea
${ }^{3}$ Yonsei Young Skin Clinic, Seoul, Korea

Received June 16, 2017

Accepted June 21, 2017

\section{Correspondence}

Dae Suk Kim

Yonsei Young Skin Clinic, 323 Eungam-ro,

Eunpyeong-gu, Seoul 03454, Korea

Tel.: +82-2-355-7582

Fax: $+82-2-355-7583$

E-mail: stone4872बanaver.com

(C) Korean Society for Laser Medicine and Surgery

(c) This is an open access article distributed under the terms of the Creative Commons Attribution NonCommercial License (http://creativecommons.org/ licenses/by-nc/4.0) which permits unrestricted noncommercial use, distribution, and reproduction in any medium, provided the original work is properly cited.
Hair graying is a common age-related hair change with tremendous social and cosmetic concerns among aged individuals. Many mechanisms contribute to hair graying, such as defective melanocyte stem cell renewal, melanocyte apoptosis, and melanocyte migration defects. Accumulation of oxidative stress has been regarded as a key factor influencing these processes. However, currently, there is no standard treatment for reversing hair graying. Herein, we report our observation of improvement of hair graying in a patient with male pattern hair loss during combined treatment with 1,927-nm fractionated thulium laser energy and intra-perifollicular polydeoxyribonucleotide injections. The patient showed increased numbers of pigmented hair follicles and clinical improvement in hair graying after 12 sessions of combined treatment. Accordingly, we suggest that the combined treatment described in this report may be a promising therapeutic method for treating hair graying.

\section{Key words}

1,927-nm fractionated thulium laser energy; Hair graying;

Polydeoxyribonucleotide; Keratinocyte growth factor 


\section{INTRODUCTION}

Hair graying can be a major concern among older adults. The average age of hair graying onset is the late30s for Asians, mid-30s for Caucasians, and mid-40s for Africans. ${ }^{1}$ Since hair color is considered important socially and cosmetically, numerous studies have sought to identify the mechanisms of hair graying. As well, various strategies have been developed to prevent or reverse hair graying. However, there is no scientific evidence supporting that any therapeutic method can prevent, halt, and reverse hair graying. In this report, we present a patient with male pattern hair loss (MPHL) who showed unexpected clinical improvement of hair graying after undergoing treatment for MPHL with a 1,927-nm fractionated thulium laser and intra-perifollicular polydeoxyribonucleotide (PDRN) injections.

\section{CASE REPORT}

A 55-year-old male patient presented with MPHL. He was willing to undergo treatments for MPHL. We planned weekly treatments with a 1,927-nm fractionated thulium laser and intra-perifollicular PDRN injections of his MPHL. The efficacy of the combined treatment for MPHL was published previously. ${ }^{2}$

After obtaining written informed consent, the patient received 12 sessions of combined treatment with a 1,927$\mathrm{nm}$ fractionated thulium laser (LASEMD ${ }^{\mathrm{TM}}$; Lutronic Corp., Goyang, Koreal and intra-perifollicular PDRN
(Placentex ${ }^{\circledR}$ integro; Mastelli Ltd., Sanremo, Italy) injections at weekly intervals. In each session, a total of about 120 shots of thulium laser energy was delivered to the scalp at a power of $3 \mathrm{~W}$ and an energy of $2 \mathrm{~mJ}$ with a static operation mode. Then, $2 \mathrm{ml}$ of PDRN was injected into the deep dermis along the frontal, mid, and vertex areas. Immediately after the treatment, a growth factor solution (LASEMD ${ }^{\mathrm{TM}}$ CK Ampoule; Lutronic Corp.) that contains keratinocyte growth factor (KGF); human cord blood cell conditioned media, which mainly contains platelet-derived growth factor, hepatocyte growth factor, insulin-like growth factor 1, and fibroblast growth factor; genistein; Scutellaria baicalensis root extract; Paeonia suffruticosa root extract; hydrolyzed sodium hyaluronate; sodium hyaluronate; mannitol; glycerin; and purified water was applied topically to the scalp.

Digital images of the scalp and hairs were obtained at $1 \mathrm{x}$ and 40x magnification using a computerized, handheld USB camera PT system (Follioscope ${ }^{\circledR}$; LeedM Corporation, Seoul, Koreal at baseline and at one week after the final treatment as described in a previous report. ${ }^{3}$ The lens of the camera was positioned at the "V" point and frontal hair line. The " $V$ " point, as described previously, refers to the vertex of the scalp where the mid-sagittal and coronal planes intersect. ${ }^{3}$ A reference point was tattooed in the frontal hair line for comparison of the hairline before and after the treatments.

As described in the previous report, the patient was satisfied with the noted clinical improvement in hair counts and hair thickness. ${ }^{2}$ In addition, the patient was also satis-
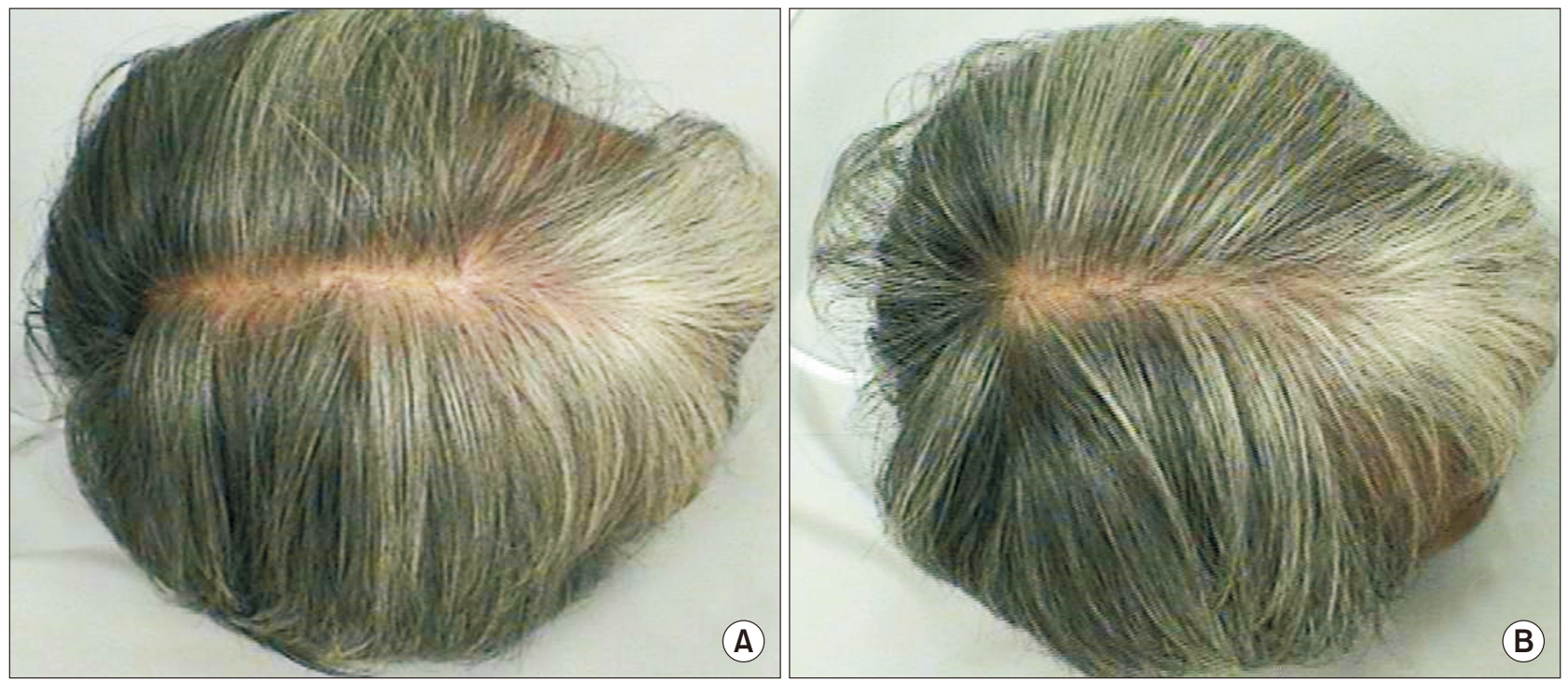

Fig. 1. Digital images of the patient's hair (A) before and (B) at one week after the last session of treatment with the thulium laser and intraperifollicular polydeoxyribonucleotide $(\mathrm{PDRN})$ injections. Original magnification $(\mathrm{A}, \mathrm{B}) \times 1$. 
fied with unexpected improvement in hair graying. The slight improvement in hair graying was observed along the frontal hair line and vertex area (Fig. 1). Improvement in hair graying was also observed in magnified views (Fig. 2). Hair graying and pigmented hair shaft counts, as well as hair counts and hair thickness, were much improved at both the "V" point (Figs. 2A, B) and the frontal hair line (Figs. 2C, D).

\section{DISCUSSION}

Hair graying is one of the most common and obvious signs of aging. It is commonly believed that, by 50 years of age, $50 \%$ of the hair in $50 \%$ of people is gray. ${ }^{4}$ As hair graying is one of the most important factors that makes people look older, numerous studies have been conducted to understand the mechanisms of hair graying. Various mechanisms were suggested for hair graying, such as melanocyte stem cells depletion, melanocyte migration defects, anagen defects, melanocyte apoptosis, and pigmentary machinery defects, all of which have been found to be related to oxidative stress. ${ }^{5}$ Melanocyte stem cells in hair are vulnerable to oxidative damage. Upon continued oxidative stress, melanocyte stem cells eventually fail to repopulate themselves, which leads to hair matrix melanocyte depletion and hair graying. ${ }^{5,6}$ The B-cell lymphoma 2 gene (BCL-2) is an anti-apoptotic oncogene that inhibits cell death, particularly in microenvironments with oxidative stress. ${ }^{7}$ As BCL-2 expression declines in aged hair follicles, melanocyte stem cells become more susceptible to oxidative stress, because of decreased protective effect from BCL-2. ${ }^{5}$

Though the mechanisms of hair graying are widely studied and hair graying is a major concern for aged people, there is currently no therapeutic method proven to prevent or reverse hair graying. Hair dyes and coloring
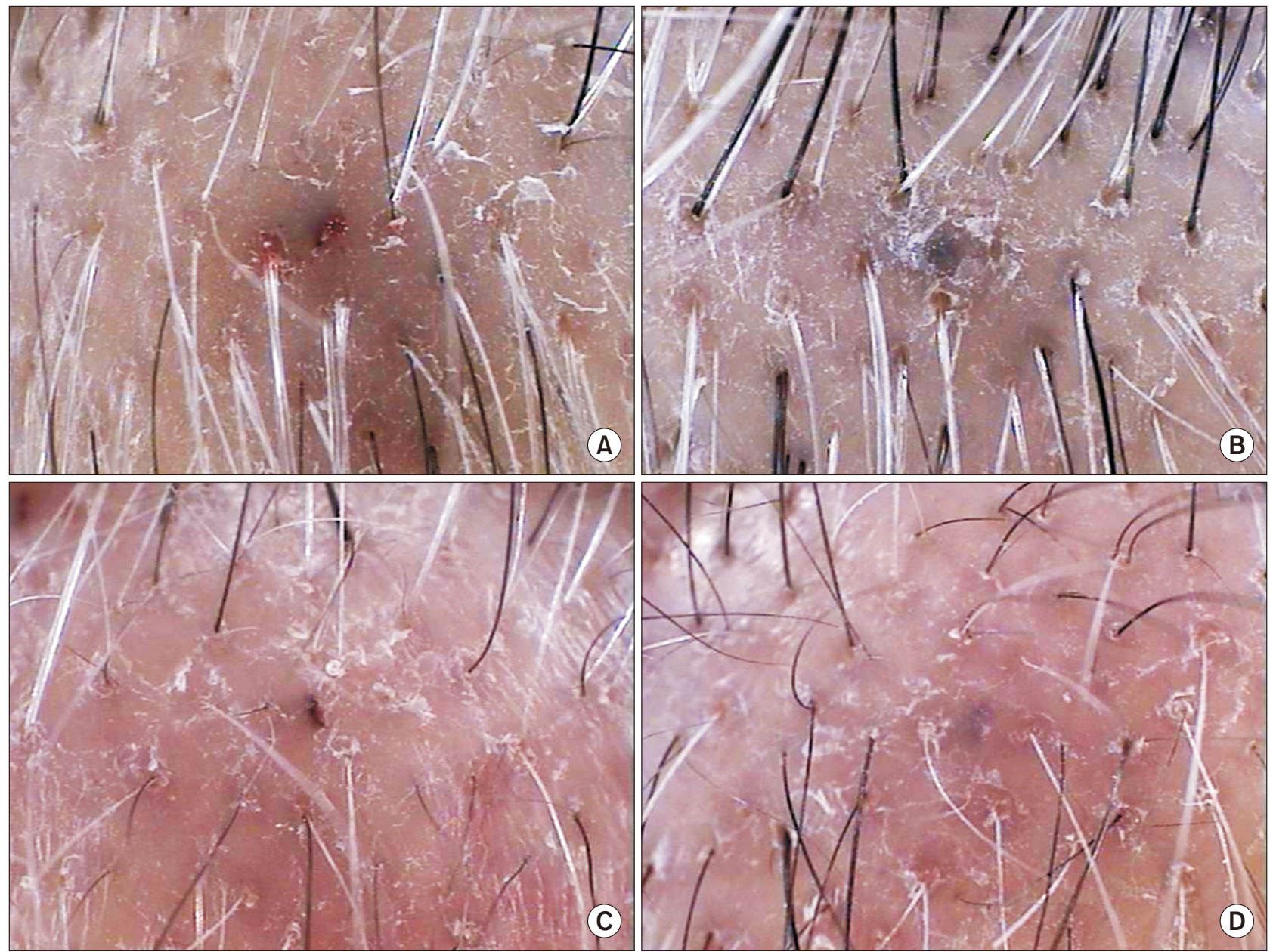

Fig. 2. Digital images of hairs at the (A, B) "V" point and (C, D) frontal hair line (A, C) before and (B, D) at one week after the last session of treatment with the thulium laser and intra-perifollicular PDRN injections. Original magnification $(A, B, C, D) \times 40$. 
are the most common way to reverse hair graying currently. Therefore, the unexpected clinical improvement in hair graying achieved using our method could shed new light on therapeutic interventions for hair graying. However, there is no scientific evidence to explain the clinical efficacy of our treatment for hair graying yet. At this point in time, we can only speculate possible mechanisms for the clinical improvement in hair graying by our method. Bitto et al. ${ }^{8}$ reported that PDRN dramatically decreased oxidative stress in an animal model of peripheral artery occlusive disease. In addition, KGF is well-known for its protective role against oxidative damage. ${ }^{9}$ One study also suggested that thulium laser energy stimulates follicular stem cells or progenitor cells to proliferate and differentiate into follicular cells. ${ }^{2}$ In our method, intra-perifollicular PDRN injections and topically applied KGF-containing growth factor solution could have exerted protective effects on melanocyte stem cells by supporting their antioxidative properties, which might have stimulated melanocyte stem cells to repopulate themselves. Overall, the thulium laser-induced tissue reactions, as well as the anti-oxidative effects of PDRN and KGF, could have supported and stimulated melanocyte stem cells to renew themselves, which eventually resulted in improvements in hair graying. Nevertheless, our report describes only the first case of clinical improvement in hair graying by combined treatment with 1,927-nm fractionated thulium laser energy, PDRN injections, and growth factor solution. Further clinical studies with a larger study population and experimental research are needed to support the effec- tiveness of our treatment method for hair graying.

\section{REFERENCES}

1. Westgate GE, Botchkareva NV, Tobin DJ. The biology of hair diversity. Int J Cosmet Sci 2013;35:329-36.

2. Cho SB, Zheng Z, Kang JS, Kim H. Therapeutic efficacy of 1,927$\mathrm{nm}$ fractionated thulium laser energy and polydeoxyribonucleotide on pattern hair loss. Med Lasers 2016;5:22-8.

3. Kang JS, Zheng Z, Choi MJ, Lee SH, Kim DY, Cho SB. The effect of CD34+ cell-containing autologous platelet-rich plasma injection on pattern hair loss: a preliminary study. J Eur Acad Dermatol Venereol 2014;28:72-9.

4. Keogh EV, Walsh RJ. Rate of greying of human hair. Nature 1965;207:877-8.

5. Seiberg M. Age-induced hair greying - the multiple effects of oxidative stress. Int J Cosmet Sci 2013;35:532-8.

6. Nishimura EK. Melanocyte stem cells: a melanocyte reservoir in hair follicles for hair and skin pigmentation. Pigment Cell Melanoma Res 2011;24:401-10.

7. Tobin DJ, Paus R. Graying: gerontobiology of the hair follicle pigmentary unit. Exp Gerontol 2001;36:29-54.

8. Bitto A, Polito F, Altavilla D, Minutoli L, Migliorato A, Squadrito F. Polydeoxyribonucleotide (PDRN) restores blood flow in an experimental model of peripheral artery occlusive disease. J Vasc Surg 2008;48:1292-300.

9. Kovacs D, Raffa S, Flori E, Aspite N, Briganti S, Cardinali G, et al. Keratinocyte growth factor down-regulates intracellular ROS production induced by UVB. J Dermatol Sci 2009;54:10613. 